\title{
DESEMPEÑO ANTE LA CORROSIÓN Y TRIBOCORROSIÓN DE ALEACIONES COMERCIALES DE ALUMINIO ANODIZADAS
}

\section{CORROSION AND TrIBOCORROSION Performance of Commercial Anodized Aluminum Alloys}

\author{
Samantha Rodríguez ${ }^{1}$, Edwuin J. Carrasquero ${ }^{2, *}$, Luis M. López ${ }^{3}$ y Jorge I. Fajardo ${ }^{3}$
}

\section{Resumen}

El presente trabajo muestra la evaluación del desempeño de dos aleaciones comerciales de aluminio anodizadas ante la corrosión y tribocorrosión. Para el anodizado se emplearon dos tipos de electrolitos distintos $\left(\mathrm{H}_{2} \mathrm{SO}_{4}\right.$ y $\left.\mathrm{H}_{2} \mathrm{C}_{2} \mathrm{O}_{4}\right)$, un valor constante de densidad de corriente y dos tiempos de anodizado (30 y 45 minutos). Usando una de las técnicas de microscopía electrónica de barrido (MEB) y espectroscopía por dispersión de la energía de rayos X (EDX) se caracterizaron las aleaciones y la morfología de las superficies formadas en las capas de óxido para las condiciones establecidas. Se encontraron diferencias notables entre la condición de anodizado (A1) a 45 minutos en $\mathrm{H}_{2} \mathrm{SO}_{4}$ para la aleación AA6061, y la condición de anodizado (D1) para el mismo tiempo en $\mathrm{H}_{2} \mathrm{C}_{2} \mathrm{O}_{4}$ para la aleación AA8011. En la condición de anodizado (A1) se encontró la formación de la morfología tipo smudge distintiva de la solución electrolítica utilizada, con un espesor promedio de capa anódica de 5,95 $\pm 0,02 \mu \mathrm{m}$, mientras que, para la condición de anodizado (D1) se obtuvo un espesor de 3,14 $\pm 0,02 \mu \mathrm{m}$, con la aparición de cavidades en toda la extensión de su superficie.

\section{Abstract}

In this work were studied two commercial anodized aluminum alloys under conditions of corrosion and tribocorrosion. For two different types of anodizing electrolyte $\left(\mathrm{H}_{2} \mathrm{SO}_{4}\right.$ and $\left.\mathrm{H}_{2} \mathrm{C}_{2} \mathrm{O}_{4}\right)$, a constant value of current density and two anodizing time (30 and 45 minutes) were used. Using techniques of Scanning Electron Microscopy (SEM) with Spectroscopy Energy Dispersive X-Ray (EDS) coupled, were characterized the alloys and morphologies of the surfaces formed in the oxide layers. Results obtained show large differences between the condition of anodized (A1) in sulfuric acid for 45 minutes in AA6061 alloy and anodized condition (D1) for the same time in oxalic acid for alloy AA8011. For sample (A1), the results reveals the formation of a morphology named "smudge" which is characteristic of the electrolytic solution used and for this case the average thickness was $5,95 \pm 0,02 \mu \mathrm{m}$. For sample (D1), the average thickness was $3,14 \pm 0,02 \mu \mathrm{m}$ and revealing the generation of cavities along all surface area.

\footnotetext{
${ }^{1}$ Facultad de Ingeniería, Universidad Central de Venezuela, Caracas - Venezuela.

${ }^{2, *}$ Facultad de Ingeniería, Escuela de Ingeniería Metalúrgica y Ciencia de los Materiales, Universidad Central de Venezuela. Facultad de Ingeniería, Caracas - Venezuela. Autor para correspondencia carrasqe@gmail.com/ edwuin.carrasquero@ucv.ve

${ }^{3}$ Grupo de Investigación en Nuevos Materiales y Procesos de Transformación (GIMAT - CIDII), Carrera de Ingeniería Mecánica, Universidad Politécnica Salesiana, Cuenca - Ecuador.
}

Recibido: 21-09-2016, aprobado tras revisión: 27-10-2016

Forma sugerida de citación: Rodríguez, S.; Carrasquero, E.; López, L. y Fajardo, J. (2016). «Desempeño ante la corrosión y tribocorrosión de aleaciones comerciales de aluminio anodizadas». InGENIUS. N. ${ }^{\circ} 16$, (Julio-Diciembre). pp. 64-72. ISSN: 1390-650X. 
Tanto para los ensayos de corrosión en cámara de niebla salina, electroquímicos de polarización potenciodinámica y tribocorrosión, se encontró que el mejor desempeño fue registrado en la condición de anodizado (A1), con una menor ganancia promedio de masa, densidad de corriente de corrosión y coeficiente de fricción. Todas las condiciones de anodizado mejoraron el comportamiento ante ensayos de degradación acelerada, en comparación con las muestras no anodizadas.

Palabras clave: anodizado, aluminio, corrosión, tribocorrosión.
Both corrosion tests in salt spray chamber, electrochemical potentiodynamic polarization and tribocorrosion found that the best behavior was obtained for sample (A1), with lower, average mass gain, corrosion current density and friction coefficient. The performance in all anodizing conditions was improved comparing against samples without anodized.

Keywords: Anodizing, Aluminum, Corrosion, Tribocorrosion 


\section{Introducción}

$\mathrm{El}$ anodizado es usado frecuentemente para proteger al aluminio de los fenómenos de desgaste y corrosión, siendo su uso principal en aplicaciones decorativas, con la posibilidad de ser coloreado en una amplia variedad de tonos. Las aleaciones de aluminio son unas de las más utilizadas a nivel industrial para la aplicación de un anodizado, aunque en una menor escala también las de titanio, tántalo y niobio son anodizados [1], [2]. Las propiedades anticorrosivas del aluminio se incrementan por el proceso de anodizado, que se basa en la técnica de oxidación anódica, donde el espesor de la capa de óxido es unos cuatro órdenes de magnitud mayor que el obtenido en los procesos de oxidación natural. La oxidación anódica del aluminio consiste en la obtención de una capa de óxido sobre la superficie metálica en el seno de un electrólito, con la participación de una fuente externa de corriente [3]. Cuando se establece un potencial entre los electrodos de la celda electrolítica, los aniones cargados negativamente migran hacia el ánodo donde se descargan con pérdida de uno o más electrones.

Los electrólitos más comunes utilizados en el proceso de anodizado se elaboran con ácido sulfúrico, pero en la industria del aluminio también se emplean otros ácidos como el crómico, fosfórico y oxálico [4].

Las características morfológicas finales de la capa anódica dependen de distintos parámetros tales como la densidad de corriente, temperatura, tiempo de inmersión, área superficial, concentración y tipo de electrolito utilizados. El anodizado mejora la capacidad de resistencia de las superficies de aluminio con respecto a las influencias externas atmosféricas y al ataque por sustancias químicas [5]. Las propiedades mecánicas de los anodizados son de gran importancia y por medio del estudio del coeficiente de fricción de la superficie anodizada se hace posible comparar de manera cualitativa cuanto es la resistencia relativa de esta capa de óxido ante la tribocorrosión [6].

Para el estudio de estos recubrimientos, se realizan ensayos de corrosión, entre los cuales se encuentran técnicas electroquímicas como los ensayos de polarización, resistencia a la polarización y potencial a circuito abierto [7], así como ensayos en cámara de niebla salina y ensayos de desgaste. Actualmente, es de especial interés conocer el efecto combinado del desgaste y la corrosión, lo cual constituye el $5 \%$ de las causas de fallos de los elementos de máquinas en el sector industrial, situándolo en el quinto puesto de los mecanismos de degradación más importantes en los términos del coste por fallo prematuro. Por estos motivos, el fenómeno de tribocorrosión ha sido foco de investigación en los últimos años, según la necesidad de seleccionar o diseñar nuevas superficies para así minimizar los costes de funcionamiento, y prolongar la vida útil del componente. Es de interés el caso particular de superficies anodizadas utilizadas en perfiles estructurales de ventanas o puertas donde pueden estar sometidas a desgaste por deslizamiento y que generen daño de la capa promoviendo el fenómeno de desgaste. El objetivo de la presente investigación es estudiar el desempeño de dos aleaciones comerciales de aluminio (AA6061 y AA8011) modificadas con distintas condiciones de anodizado, y su evaluación mediante ensayos de resistencia a la corrosión y tribocorrosión.

Usando una de las técnicas de microscopia electrónica de barrido (MEB) con espectroscopia por dispersión en la energía de rayos (EDX) se caracterizaron las morfologías superficiales de las capas de óxido, con el fin de conocer el comportamiento de estas aleaciones de aluminio, que son ampliamente utilizadas en la industria en diversas aplicaciones, sometiéndolas a condiciones y mecanismos de desgaste corrosivos altamente degradantes.

\section{Experimental}

Las aleaciones de aluminio utilizadas en este estudio fueron láminas de dimensiones de $20 \times 150 \times 1 \mathrm{~mm}$ de aluminio comercial AA6061 y AA8011. En la Tabla 1 se muestra la composición química nominal respectivas.

Tabla 1. Composición química nominal de las aleaciones de aluminio AA6061 y AA8011 [8]

\begin{tabular}{cccccc}
\hline $\begin{array}{c}\text { Contenido } \\
(\%)\end{array}$ & Si & Fe & $\mathbf{C u}$ & $\mathbf{M g}$ & $\mathbf{M n}$ \\
\hline AA6061 & $0,40-0,80$ & 0,7 & $0,15-0,40$ & $0,80-1,20$ & - \\
AA8011 & $0,50-0,90$ & $0,60-1,00$ & 0,10 máx. & 0,05 máx. & 0,20 máx. \\
\hline
\end{tabular}

Para la caracterización microestructural de estas aleaciones se realizó en primer lugar, una preparación metalográfica, posteriormente, fueron atacadas con una solución de ácido fluorhídrico al $2 \% \mathrm{v} / \mathrm{v}$ para el revelado de las fases presentes en la microestructura. El proceso de anodizado se realizó para cada tipo de aleación bajo distintas condiciones experimentales presentadas en la Tabla 2.

Tabla 2. Matriz experimental del proceso de anodizado

\begin{tabular}{cccc}
\hline Electrólito & Aleación & $\begin{array}{c}\text { Tiempo } \\
\text { (min) }\end{array}$ & Identificación \\
\hline \multirow{2}{*}{ Ácido sulfúrico } & AA6061 & 45 & $\mathrm{~A} 1$ \\
(H2SO4) & & 30 & $\mathrm{~A} 2$ \\
& $\mathrm{~A} 48011$ & 45 & $\mathrm{~B} 1$ \\
& & 30 & $\mathrm{~B} 2$ \\
Ácido oxálico & \multirow{2}{*}{ AA6061 } & 45 & $\mathrm{C} 1$ \\
(H2C2O4) & \multirow{2}{*}{$\mathrm{AA} 8011$} & 45 & $\mathrm{C} 2$ \\
& & 30 & $\mathrm{D} 1$ \\
\hline
\end{tabular}

Se emplearon dos electrolitos distintos: ácido sulfúrico $\left(\mathrm{H}_{2} \mathrm{SO}_{4}\right)$ con una concentración al 
$18 \%$ en v/v y ácido oxálico $\left(\mathrm{H}_{2} \mathrm{C}_{2} \mathrm{O}_{4}\right)$ con una concentración de $40 \mathrm{~g} / \mathrm{l}$. Se fijaron dos valores de tiempo de inmersión 30 y 45 min, a temperatura ambiente $\left(26 \pm 2{ }^{\circ} \mathrm{C}\right)$. La densidad de corriente utilizada fue de $1,2 \mathrm{~A} / \mathrm{dm}^{2}$, utilizando como cátodo láminas de acero inoxidable de dimensiones $20 \times 200 \times 1 \mathrm{~mm}$. Cabe destacar que el proceso de anodización fue realizado en disposición de celdas conectadas en serie, estableciendo valores de voltaje entre 35-40 V. Antes del proceso de anodizado se realizó la preparación superficial de las muestras, en primer lugar se desengrasaron sumergiéndolas durante 5 min en una disolución acuosa de ácidos fosfórico y crómico en concentraciones de 250 y $55 \mathrm{~g} / \mathrm{l}$, respectivamente; a una temperatura de $35{ }^{\circ} \mathrm{C}$.

Posteriormente, se decaparon en una solución acuosa de hidróxido de sodio de $100 \mathrm{~g} / \mathrm{l}$ a $45{ }^{\circ} \mathrm{C}$ durante $60 \mathrm{~s}$ y se neutralizaron por inmersión durante unos segundos en la misma disolución usada para desengrasar. Para finalizar el proceso de anodizado, a las probetas se les aplicó un sellado hidrotérmico, en agua desionizada a la temperatura de ebullición, durante $60 \mathrm{~min}$, por ser un tiempo suficiente para conseguir los índices de calidad reportados en [9]. Se realizaron los ensayos en cámara de niebla salina según lo establecido en la norma ASTM B117 [10], por duplicado para cada condición, a través de un equipo marca HARSHAW modelo 22 , a una temperatura de $35 \pm 1{ }^{\circ} \mathrm{C}$ y tiempo de exposición de $120 \mathrm{~h}$.

Se efectuaron ensayos electroquímicos de polarización potenciodinámica con un equipo potenciostato/galvanostato marca CM Instruments, modelo GillAC, según la norma ASTM G59-97 [11], disponiendo la celda electroquímica de un electrodo de referencia de calomelano saturado (SCE) y un contra electrodo de grafito, los ensayos se realizaron a temperatura ambiente en solución salina al 3,5\% p/p de $\mathrm{NaCl}$, empleando un barrido de potencial desde $-400 \mathrm{mV}$ hasta $1500 \mathrm{mV}$ a una velocidad de $1 \mathrm{mV} / \mathrm{s}$. Los ensayos de tribocorrosión se realizaron a temperatura ambiente de acuerdo con la norma ASTM G119 [12], haciendo uso de un tribómetro marca MicroTest modelo MT4002, bajo la configuración bola sobre disco, con una carga normal $2 \mathrm{~N}$, una distancia de deslizamiento de $125 \mathrm{~m}$, un radio de contacto de $5 \mathrm{~mm}$, velocidad de rotación de 100 r. p. m. y utilizando como contraparte bolas de $6 \mathrm{~mm}$ de diámetro de alúmina.

Este tribómetro tiene acoplado un potenciostato marca AMEL Instruments modelo 2049, que emplea un electrodo de referencia $\mathrm{Ag} / \mathrm{AgCl}$ y un contra electrodo de grafito, se empleó la misma solución de $\mathrm{NaCl}$ de los ensayos potenciodinámicos. En estos ensayos sinérgicos, se destaca la inducción de corriente cero, con la medición del potencial libre de corrosión de cada muestra ensayada. Finalmente, a través del uso de un microscopio óptico marca Olympus y de un microscopio electrónico de barrido (MEB) marca Hitachi
S-2400 con un espectrómetro de por dispersión en la energía de rayos-X (EDX), se realizó la medición de los espesores a través de la sección transversal de las muestras, se caracterizaron las aleaciones y la morfología de las superficies formadas en las capas de óxido para las condiciones establecidas, se identificaron los productos de corrosión y tribocorrosión en las superficies de las muestras.

\section{Resultados y discusión}

En las Figuras 1a y 1b se presentan las fotomicrografías obtenidas por MEB correspondientes a la caracterización microestructural de las aleaciones de aluminio sin anodizar AA6061 y AA8011, respectivamente. En ambas figuras se corroboró por análisis puntuales por EDX la presencia de fases intermetálicas, las cuales están uniformemente distribuidas en la matriz, estas fases señaladas como 1 y 2 en la Figura 1a corresponden a fases ricas en Si y Mg, respectivamente. Mientras que, en la Figura 1b para la aleación AA8011 las fases indicadas como 1 refieren a una fase rica en Fe.
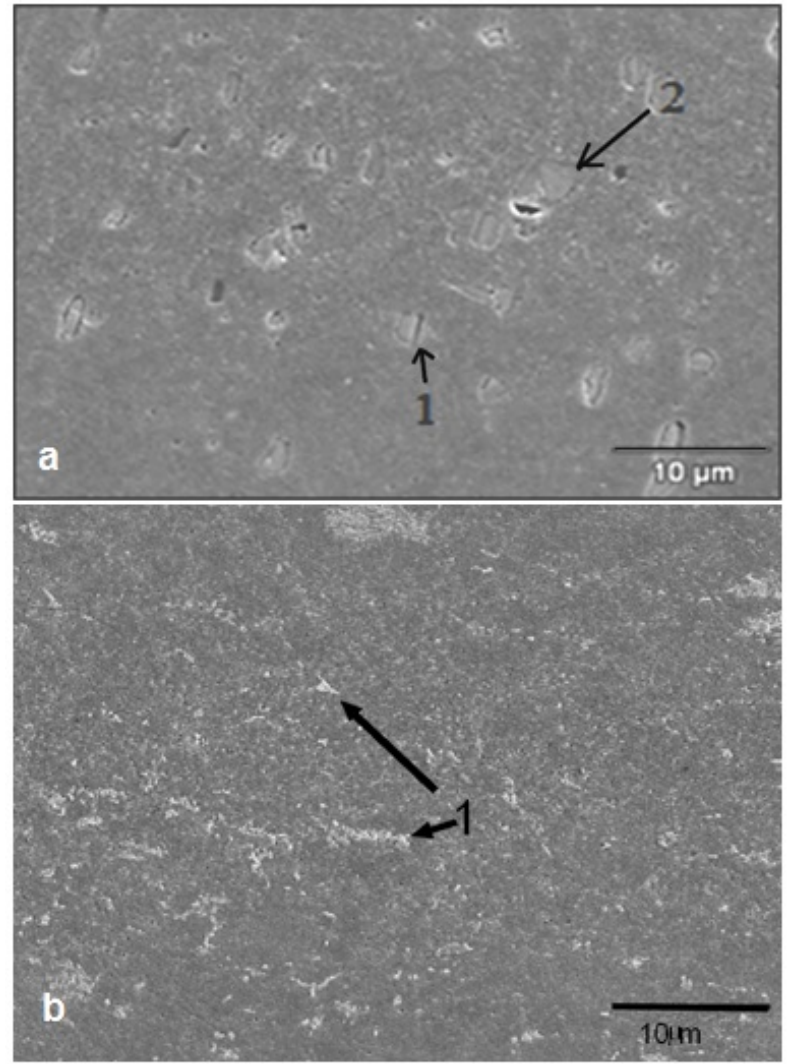

Figura 1. Fotomicrografía de la microestructura: a) AA6061; b) AA8011. MEB-SE (1000X)

En la Tabla 3, se indica los valores promedios de los espesores obtenidos para cada una de las condiciones de anodizado, para el mayor tiempo de inmersión (45 min). Se determinó que el mayor espesor de la capa 
anódica se logró con un mayor tiempo de anodización (45 min) para todas las condiciones sometidas al proceso.

Tabla 3. Espesores promedios de las capas anodizadas

\begin{tabular}{cc}
\hline Condición & Espesor promedio $(\mu \mathrm{m}) \cdot$ \\
\hline A1 & $5,95 \pm 0,02$ \\
B1 & $1,31 \pm 0,02$ \\
C1 & $4,27 \pm 0,02$ \\
D1 & $3,14 \pm 0,02$ \\
\hline
\end{tabular}

Los mayores valores promedio de espesor de la capa anódica, correspondieron a las muestras anodizadas de AA6061 obtenidas para ambos electrolitos, siendo $40 \%$ mayor en ácido sulfúrico en comparación al oxálico, en caso contrario para la AA8011 los mayores espesores encontrados correspondieron cuando se utilizó como electrolito el ácido oxálico, obteniéndose un incremento del $140 \%$ con respecto al sulfúrico. Aunque en algunas condiciones no se alcanzaron los valores de espesor de capa anódica esperados, en la mayoría de las condiciones establecidas se obtuvieron valores cercanos a los adecuados de recubrimiento anódico en correspondencia con el rango establecido [13] de bajas densidades de corriente y para el número de muestras anodizadas en serie.

En la Figura 2, se muestra las superficies y secciones transversales de las probetas anodizadas para las distintas condiciones experimentales.

Como se puede observar en esta figura se encontró diferencias marcadas en la morfología superficial entre la condición de anodizado (A1) para la aleación AA6061 en ácido sulfúrico durante 45 minutos y la condición de anodizado (C1) para esta misma aleación en ácido oxálico a igual tiempo. Para la condición de anodizado (A1) (Figura 2a y 2b), se encontró una estructura propia del anodizado en ácido sulfúrico, correspondiente a cristales de diversas características y tamaños, conocida en la literatura como tipo smudge originada por la precipitación del aluminio durante la reacción de sellado hidrotérmico [14].

A través de los análisis puntuales por EDX realizados para esta condición, se registró la presencia de una mayor relación en concentración atómica porcentual de $\mathrm{Al}$ y $\mathrm{O}$ en comparación con los restantes análisis por EDX obtenidos, lo cual puede relacionarse con la preponderante interacción entre el agua y la pared de las celdas con una posible formación de bayerita $\left(\mathrm{Al}_{2} \mathrm{O}_{3} \cdot 3 \mathrm{H}_{2} \mathrm{O}\right.$ [1], registrándose también la presencia de azufre proveniente de la contaminación del ácido sulfúrico. No obstante, para la condición de anodizado (D1), se obtuvo una menor relación Al-O, indicativo de la deficiente formación de la capa anódica sobre el sustrato.
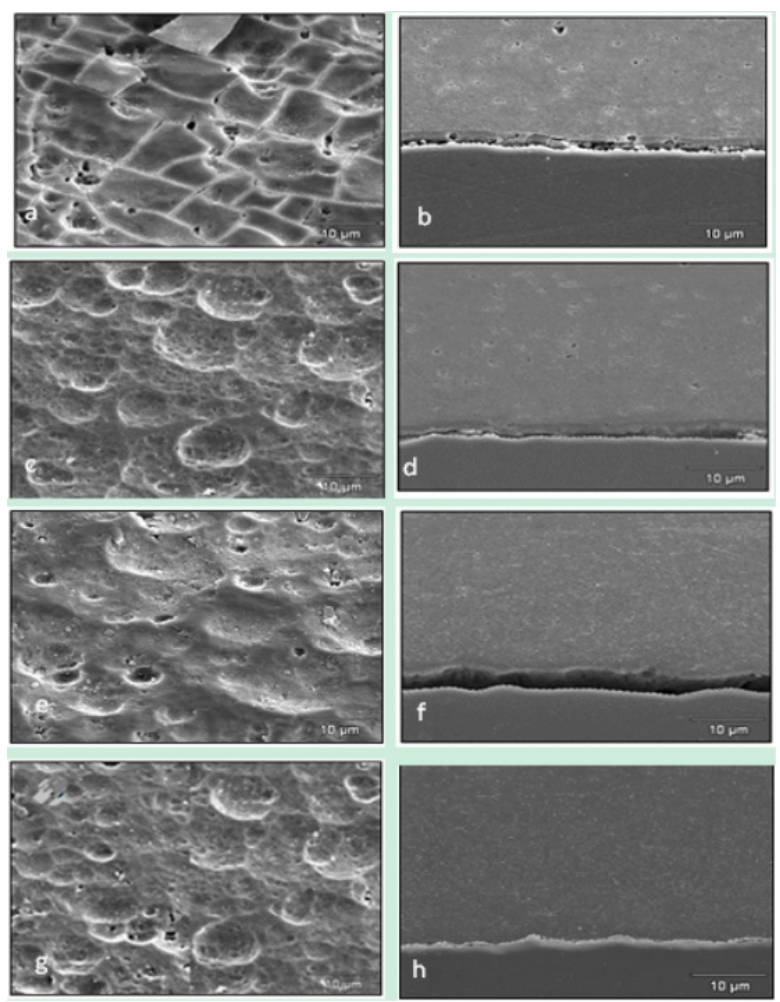

Figura 2. Fotomicrografía por MEB. (1000 X) de las superficies y secciones transversales de las muestras anodizada: (a y b) Condición A1; (c y d) Condición B1; (e y f) Condición C1; (g y h) Condición D1. (Tiempo: 45 min)

En general, la apariencia de las superficies anodizadas para esta condición es heterogénea con la presencia de microcavidades, lo cual es indicativo de una condición no fue eficiente el sellado hidrotérmico. No se observó la característica morfología de crecimiento en columnas de celdillas hexagonales por ser esta más común en recubrimientos no sellados. Según se evidenció en las fotomicrografías de las secciones transversales las capas anódicas más homogéneas y con mejor adherencia con el sustrato correspondió a la AA8011 en ambos tipos de electrolito (Figura $2 \mathrm{~d}$ y $2 \mathrm{~h}$ ).

Sin embargo, el mayor espesor del recubrimiento anódico, observado en la sección transversal de las muestras, corresponde a la aleación AA6061, en comparación a la aleación AA8011; esto puede deberse a la conductividad de los iones presentes por parte de la primera aleación y los precipitados presentes en ella que contribuyen a la formación de la película de óxido a través del proceso de anodizado. De acuerdo con estos resultados se evaluaron su resistencia a la corrosión y tribocorrosión solo las muestras anodizadas de mayor espesor (condición (A1) y (D1)) y compararlas con respecto a ambas aleaciones sin anodizar.

En la Figura 3, se exhiben los valores de ganancia de masa de las muestras anodizadas y para los sustratos sin anodizar, correspondientes a los ensayos en cámara de niebla salina, mientras que las fotomicro- 
grafías por MEB de la morfología de las superficies expuestas a este ensayo se muestran en la Figura 4.

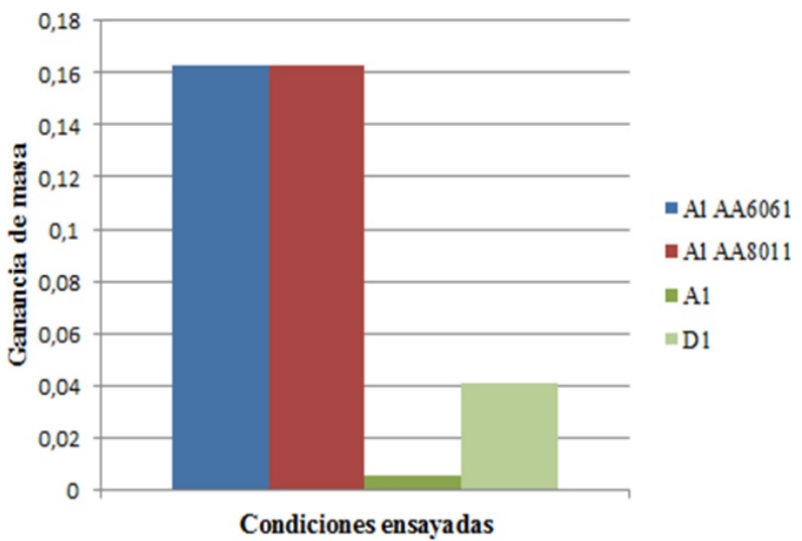

Figura 3. Ganancia de masa promedio en cámara de niebla salina para las muestras ensayadas
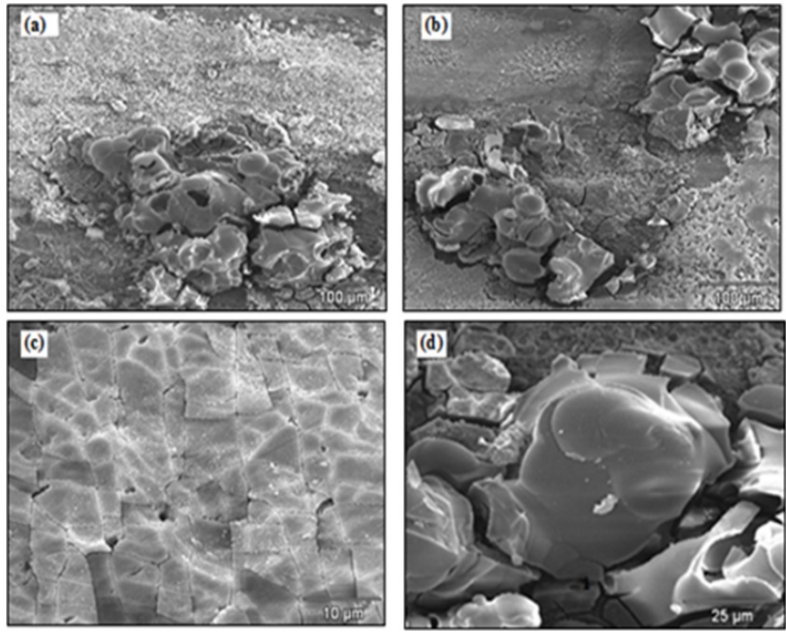

Figura 4. Fotomicrografías superficiales de las muestras ensayadas en cámara de niebla salina. (a) AA6061 sin anodizar, (b) AA8011 sin anodizar, (c) A1, (d) D1

Los valores potencial de corrosión (Ecorr), densidad de corriente de corrosión (Icorr) y resistencia a la polarización (Rp) obtenidos de los ensayos electroquímicos de polarización potenciodinámica, se indican en la Tabla 4.

Tabla 4. Potencial de corrosión, densidad de corriente de corrosión, y resistencia a la polarización de los sustratos y muestras anodizadas

\begin{tabular}{cccc}
\hline Identificación & Ecorr $(\mathbf{m V})$ & Icorr $\left(\mu \mathbf{A} / \mathbf{c m}^{2}\right)$ & $\mathbf{R p}\left(\Omega \cdot \mathbf{c m}^{2}\right)$ \\
\hline AA6061 & $-809,17$ & 379,28 & 8,39 \\
AA8011 & $-995,29$ & 337,43 & 10,61 \\
Condición A1 & $-805,84$ & 110,12 & 160,16 \\
Condición D1 & $-952,25$ & 302,12 & 12,17 \\
\hline
\end{tabular}

En cuanto los sustratos, los valores de Icorr para ambas aleaciones son similares y mayores. Por MEB se identificó en ambas aleaciones un mecanismo de corrosión generalizada, sin rastro de la superficie original de cada una, lo que corrobora la notable disolución del sustrato, el cual inicia en la corrosión localizada asociada a un mecanismo de ruptura de la película de óxido natural y el ingreso de los iones cloruro provenientes de la solución salina. Con respecto a la condición (A1), se observa una mejoría sobresaliente en cuanto a resistencia a la corrosión se refiere, en comparación con los valores obtenidos para el sustrato, lo cual se verifica con la disminución de la velocidad de corrosión en un $50 \%$ aproximadamente, y con el mayor valor de $\mathrm{Rp}$.

Para esta condición de anodizado, la capa de óxido formada proporcionó resistencia adecuada a la corrosión electroquímica como fue reportado por Li et al., [7] para condiciones de deposición similares, aunque se puede notar signos de corrosión superficial en algunas zonas, no tan incidente en comparación a la establecida en el sustrato, con la presencia de picaduras y grietas en algunas regiones con atrapamiento de azufre. Con respecto a la condición (D1), se pudo notar un menor desempeño contra la corrosión, lo cual se corrobora por un valor de Icorr semejante al obtenido para el sustrato (AA8011), así como también por el grave deterioro superficial presentado, el cual se debe a una mayor facilidad en la salida de iones $\mathrm{Al}^{+3}$, y un relativo fácil ingreso de los iones $\mathrm{O}^{-2}$ en el proceso de formación de la capa anódica, lo que origina que no se alcance un espesor, y características protectoras adecuadas, así como también a la porosidad presente en esta condición de anodizado y cuyo mecanismo fue reportado por Suay et al., [15].

En la Figura 5, se exponen las curvas pertinentes al coeficiente de fricción (COF) y potencial a circuito abierto en función de la distancia de deslizamiento de las muestras sometidas a ensayos de tribocorrosión, los resultados obtenidos de estos ensayos se resumen en la Tabla 5.

Tabla 5. Resultados de los ensayos de tribocorrosión de los sustratos y muestras anodizadas

\begin{tabular}{cccc}
\hline Identificación & $\mathbf{D}(\mathbf{m})^{*}$ & $\mathbf{E p f}(\mathbf{m V})^{* *}$ & $\begin{array}{c}\text { Coeficiente } \\
\text { de fricción } \\
\text { (COF) }\end{array}$ \\
\hline AA6061 & 0,59 & $-1073,12 \pm 1,02$ & $0,82 \pm 0,04$ \\
AA8011 & 0,53 & $-1027,93 \pm 1,12$ & $0,91 \pm 0,03$ \\
Condición A1 & 0,62 & $-1300,43 \pm 2,78$ & $0,88 \pm 0,01$ \\
Condición D1 & 20,31 & $-1285,65 \pm 2,19$ & $0,64 \pm 0,01$ \\
\hline
\end{tabular}

En cuanto a las muestras sin anodizar, se observó por MEB en las huellas de desgaste que para ambas aleaciones las características morfológicas son similares entre sí, con la formación de una capa compacta de partículas de desgaste sobre ellas, donde es evidente el desgaste adhesivo con un posterior desprendimiento, y formación de partículas de debris entre el sustrato y la contraparte. 
Asimismo, para los sustratos se registró la caída de potencial libre de corrosión (Epf) con el aumento del coeficiente de fricción el cual ocurrió a los pocos metros de deslizamiento del ensayo aprox. 0,5 m (Figura 5a y $5 b$ ), donde la capa natural de óxido es desprendida en la dirección del deslizamiento, registrándose fluctuaciones bruscas del (COF) la para la aleación AA8011 (Figura 5b), lo cual se infiere es la tendencia de las muestras a la regeneración de la capa pasiva natural, aunque no se completa por la acción consecutiva, y conjunta de los fenómenos de desgaste-corrosión.
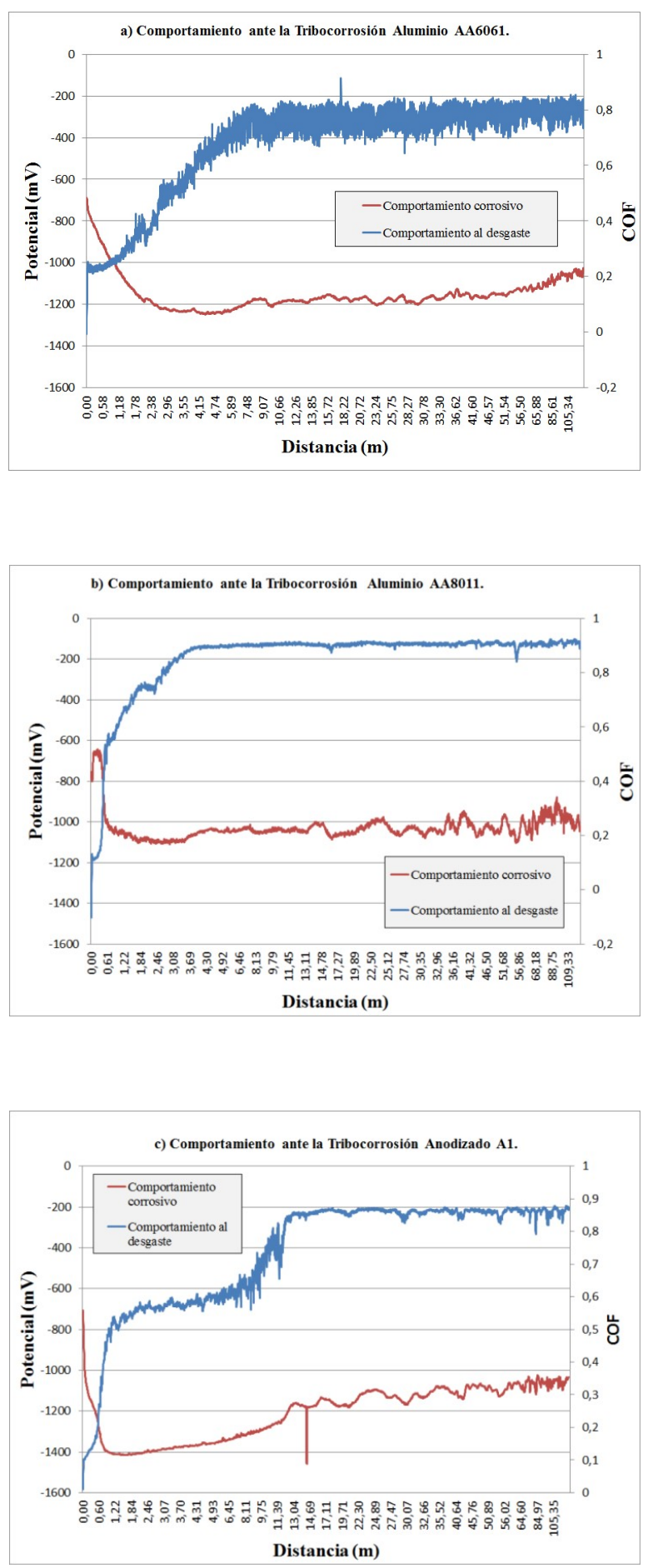

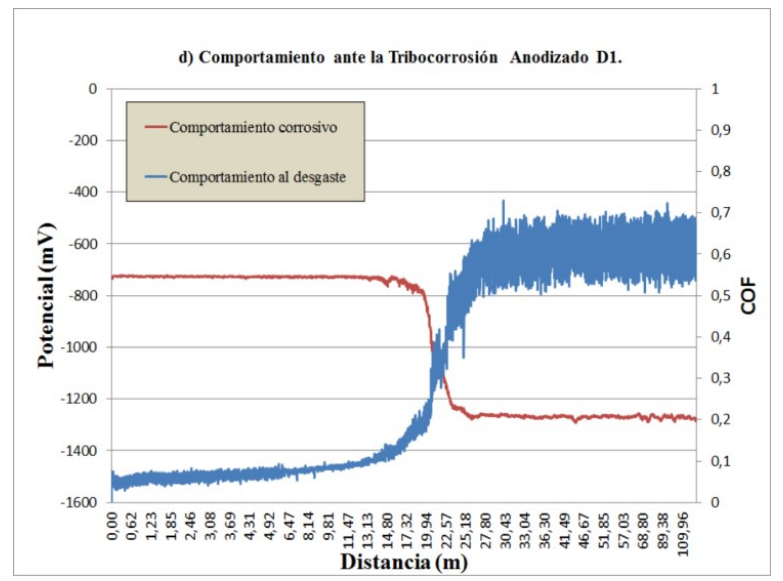

Figura 5. Coeficiente de fricción y potencial a circuito abierto, en función de la distancia de deslizamiento, de las muestras ensayadas en tribocorrosión. (a) AA6061; (b) AA8011; (c) A1; (d) D1

Para la condición de anodizado (A1), se evidenció por MEB la presencia de un desgaste adhesivo, con la delaminación de las asperezas de menor dureza, por la acción de la fuerza tangencial ejercida en los puntos de contacto, lo cual induce la deformación plástica superficial y subsuperficial, con la presencia importante de grietas.

Se nota un comportamiento similar en las curvas obtenidas para esta condición, en comparación con los sustratos ensayados, con la caída brusca del potencial a circuito abierto, y en el mismo punto, el cambio en aumento del COF, lo cual es indicativo de la poca resistencia ante fenómenos tribocorrosivos, que presenta esta condición de anodizado, donde también se observa un comportamiento altamente activo de las muestras, con una tendencia progresiva de aumento del potencial libre de corrosión, lo cual en conjunto con los picos presentados, se puede inferir una posible regeneración de la capa pasiva natural del material.

Para la condición de anodizado (D1), se identificaron por MEB la presencia de grietas pequeñas en algunas zonas de la huella de desgaste, con la aparición de un mecanismo mixto de desgaste de abrasión preponderante en el centro de la huella, y en su periferia la delaminación de las asperezas formadas.

Asimismo, en esta condición, se observa en la Figura 5d un cambio en el comportamiento con respecto a las otras condiciones ensayadas, ya que se obtuvo el rompimiento de la capa anódica a una distancia de ensayo mayor (aprox. $21 \mathrm{~m}$ ), lo cual se indica por el decrecimiento del potencial libre de corrosión, y el aumento brusco COF, donde es notable la fluctuación en los valores registrados, esto es debido a la interacción cíclica entre los debris generados atrapados en el área de contacto del par tribológico.

Aun cuando para esta condición de anodizado (D1) presentó un menor espesor de capa anódica con la 
presencia de microcavidades y menor resistencia a la corrosión, es en este caso, la que presenta mejor comportamiento contra fenómenos de acción conjunta desgaste-corrosión, deduciéndose que la adherencia presentada por esta condición es eficiente, complementado con el hecho de la menor presencia de grietas en la capa anódica establecida.

En general, todas las condiciones ensayadas, tanto los sustratos sin anodizar y con recubrimiento anódico, fueron gobernadas por un mecanismo de desgaste abrasivo severo; en algunas zonas hubo desgaste adhesivo con indicios de deformación plástica. Con respecto al fenómeno de corrosión presente en las distintas muestras, se atribuye al atrapamiento de los iones cloruro provenientes de la solución salina en distintas zonas de las superficies, donde el deterioro se encuentra asociado a una difusión del oxígeno disuelto en el medio a la superficie, cuando la solución se encuentra en movimiento, facilitando los procesos catódicos por el transporte de oxígeno en el proceso.

\section{Conclusiones}

En función de los resultados obtenidos de los diferentes ensayos de resistencia a la corrosión y tribocorrosión para las dos aleaciones comerciales de aluminio (AA6061 y AA8011) modificadas con distintas condiciones de anodizado se concluye lo siguiente:

- El mejor tiempo de deposición donde se alcanza los mayores espesores de capa anódica corresponde a $45 \mathrm{~min}$.

- Las muestras presentan porosidad superficial, con la creación de microcavidades donde se produce la deposición de productos corrosivos. Los sustratos presentan corrosión generalizada, con la formación de una capa gruesa de productos de corrosión, así como atrapamiento de iones Cl-, bajo los distintos ensayos realizados.

- La AA6061 anodizada en ácido sulfúrico, presenta morfología de cristales smudge, así como el mayor espesor promedio de capa anódica correspondiente al bajo valor de densidad de corriente utilizada, con un buen comportamiento en ensayos de cámara de niebla salina y polarización potenciodinámica, aunque presenta deficiente comportamiento ante fenómenos tribocorrosivos, por la presencia de grietas superficiales.

- La AA8011 anodizada en ácido oxálico presenta el menor espesor promedio de capa anódica, con un mejor comportamiento ante la acción conjunta desgaste-corrosión, por la calidad en adherencia del recubrimiento.
- En general, las muestras anodizadas mejoran el comportamiento contra la corrosión acelerada y tribocorrosión, en comparación con las muestras sin anodizar.

\section{Agradecimiento}

Al Proyecto FONACIT PEI-2011001094 Mejoras de la resistencia a la corrosión de aleaciones de aluminio de producción nacional mediante la aplicación de un proceso de anodizado por el financiamiento recibido para el desarrollo de esta investigación y al Departamento de Ciencia de los Materiales de la Universidad Simón Bolívar, por la colaboración recibida para los ensayos de tribocorrosión.

\section{Referencias}

[1] M. J. Bartolomé, S. Feliu Jr., V. López, E. Escudero, J. A. González, , and S. Feliu, "Efecto de las heterogeneidades superficiales de las aleaciones de aluminio sobre el crecimiento y propiedades de las capas anódicas," Rev. Metal, vol. 43, no. 4, pp. 252-265, Barcelona, 2000.

[2] L. E. V. Guillén, "Estudio del proceso de anodizado en aluminio 3003 y 6063," EAE, 2012.

[3] G. E. Thompson, Y. Xu, P. Skeldon, K. Shimizu, S. H. Han, and G. C. Wood, "Anodic oxidation of aluminium," Philos. Mag. B, vol. 55, no. 6, pp. 651-667, 1987.

[4] E. Álvarez-Ayuso, "Approaches for the treatment of waste streams of the aluminium anodizing industry," J. Hazard. Mater, vol. 164, no. 2, pp. 409-414, 2009.

[5] M. J. Bartolomé, J. F. del Río, E. Escudero, S. Feliu, V. López, E. Otero, and J. A. González, "Behaviour of different bare and anodized aluminium alloys in the atmosphere," Surf. Coat. Technol., vol. 202, no. 12, pp. 2783-2793, 2008.

[6] R. Jamaati, M. R. Toroghinejad, J. A. Szpunar, and D. Li, "Tribocorrosion behavior of aluminum/alumina composite manufactured by anodizing and arb processes," J. Mater. Eng. Perform, vol. 20, no. 9, pp. 1600-1605, 2011.

[7] X. Li, X. Nie, L. Wang, and D. O. Northwood, "Corrosion protection properties of anodic oxide coatings on an al-si alloy," Surf. Coat. Technol., vol. 200, no. 5, pp. 1994-2000, 2005.

[8] J. R. Davis, J. R. Davis, and others., "Aluminum and aluminum alloys." ASM International, 1993. 
[9] A. Bautista, R. Lizarbe, E. Otero, V. López, and J. A. González, "Nuevas alternativas a los métodos industrialmente implantados para el sellado del aluminio anodizado," Rev. Metal., vol. 35, no. 3, pp. 195-202, 1999.

[10] A. International, "Astm b-117, standard practice for operating salt spray (fog) apparatus," ASTM Int. 1997 Ed., 1997.

[11] G. ASTM, "Standard test method for conducting potentiodynamic polarization resistance measurements." 2009.

[12] B. W. Madsen, "Standard guide for determining amount of synergism between wear and corrosion,"
ASTM G119-93 Annu. Book ASTM Stand., vol. 3, pp. 507-512, 1994.

[13] J. L. Gazapo and J. Gea, "Anodizing of aluminium," TALAT Lect., vol. 5203, 1989.

[14] C. V. Hernández, F. N. Jiménez, and C. L. L. Calderón, "Crecimiento de películas de óxido de aluminio por métodos electroquímicos," Sci. Tech., vol. 1, no. 34, 2007.

[15] J. J. Suay, E. Giménez, T. Rodríguez, K. Habbib, and J. J. Saura, "Characterization of anodized and sealed aluminium by eis," Corros. Sci., vol. 45, no. 3, pp. 611-624, 2003. 